\title{
CRESCIMENTO DE MUDAS DE GONÇALO-ALVES (Astronium fraxinifolium) SOB DIFERENTES FONTES E DOSES DE NITROGÊNIO ${ }^{1}$
}

Diego Gonçalves Feitosa², Kátia Luciene Maltoni ${ }^{3}$, Ana Maria Rodrigues Cassiolato ${ }^{3}$ e Monica Orlandi Paiano ${ }^{4}$

\begin{abstract}
RESUMO - A degradação do cerrado e a perda de sua biodiversidade demandam a revegetação de áreas desse bioma, onde existem espécies com potencial de uso econômico e ambiental pouco conhecidos. Este trabalho teve o objetivo de avaliar o efeito de diferentes fontes e doses de nitrogênio sobre o crescimento de mudas de Gonçalo-Alves (Astronium fraxinifolium), espécie arbórea nativa do cerrado ameaçada de extinção. O experimento foi realizado em estufa na UNESP/Campus de Ilha Solteira. O solo utilizado (Latossolo Vermelho) foi coletado na profundidade de 0,0 a 0,20 m, em Selvíria (MS). As fontes de nitrogênio utilizadas $\left(\left(\mathrm{NH}_{4}\right)_{2} \mathrm{SO}_{4}, \mathrm{NH}_{4} \mathrm{NO}_{3}\right.$ e Ca( $\left.\left(\mathrm{NO}_{3}\right)_{2}\right)$ foram aplicadas nas doses de 0; 50; 100; 150 e $200 \mathrm{mg} \mathrm{dm}^{-3}$. O N aplicado na forma de $\mathrm{Ca}\left(\mathrm{NO}_{3}\right)_{2}$ apresentou melhor resultado para o solo utilizado. O crescimento das mudas de Gonçalo-Alves não foi influenciado pelas diferentes fontes de $\mathrm{N}$, mas respondeu às doses. A altura da planta, o diâmetro do coleto, o teor de clorofila foliar, o peso da matéria fresca e seca da parte aérea e do sistema radicular, o peso seco total, bem como o IQD e a razão entre o peso da matéria seca da parte aérea e do sistema radicular, apresentaram valor máximo

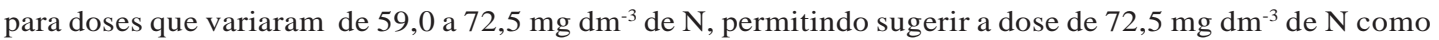
suficiente para o crescimento satisfatório de mudas de Gonçalo-Alves.
\end{abstract}

Palavras-chave: Cerrado, Área degradada e Reflorestamento.

\section{GONÇALO ALVES' (Astronium fraxinifolium) SEEDLINGS GROWTH AT DIFFERENT NITROGEN SOURCES AND LEVELS}

\begin{abstract}
Degradation of cerrado and the loss of its biodiversity require the revegetation of the areas in this biome, where there are species with little known potential of economical and environmental use. The objective of this work was to evaluate the effect of different sources and levels of nitrogen on growth of seedlings of Gonçalo-Alves(Astronium fraxinifolium), tree species native from cerrado, threatened of extinction. The experiment was carried out in a greenhouse, at UNESP/Campus of Ilha Solteira. The soil used (Red Latosol) was collected from 0.0 to $0.20 \mathrm{~m}$ layer in Selvíria (MS). The utilized sources of $\mathrm{N}$, $\left(\mathrm{NH}_{4}\right)_{2} \mathrm{SO}_{4}, \mathrm{NH}_{4} \mathrm{NO}_{3}$ and $\left.\mathrm{Ca}\left(\mathrm{NO}_{3}\right)_{2}\right)$, were applied at doses of $0 ; 50 ; 100 ; 150$ and $200 \mathrm{mg} \mathrm{dm}^{-3}$. The $\mathrm{N}$ applied as $\mathrm{Ca}\left(\mathrm{NO}_{3}\right)_{2}$ presented the best results for the soil used. Gonçalo-Alves seedlings growth was not influenced by the $N$ sources, but it responded to the doses of $N$. Height, stem diameter, content of leaf chlorophyll, fresh and dry matter weights of the aerial section, weights of fresh and dry matter of root system, total dry matter weight, as well as IQD and ratio of weight of dry matter of aerial section and root system presented maximum value for doses which ranged from 59.0 to $72.5 \mathrm{mg} \mathrm{dm}^{-3} \mathrm{~N}$, suggesting the dose of $72.5 \mathrm{mg} \mathrm{dm}^{-3}$, as sufficient for a satisfactory growth of seedlings of Gonçalo Alves.
\end{abstract}

Keywords: Cerrado, Degraded area and Reforestation.

\footnotetext{
${ }^{1}$ Recebido em 27.02.2009 e aceito para publicação em 14.04.2011.

${ }^{2}$ Graduação em agronomia pela Universidade Estadual Paulista Júlio de Mesquita Filho, UNESP, Brasil. E-mail: <diegogfeitosa@yahoo.com.br>.

${ }^{3}$ Universidade Estadual Paulista Júlio de Mesquita Filho, UNESP, Brasil. E-mail: <maltoni@agr.feis.unesp.br> e <anamaria@bio.feis.unesp.br>.

${ }^{4}$ Mestrado em andamento em MICROBIOLOGIA pelo IBILCE - Instituto de Biociências, Letras e Ciências exatas. E-mail: $<$ monica_paiano@hotmail.com>.
} 


\section{INTRODUÇÃO}

O cerrado, um dos mais importantes biomas brasileiros, vem sendo intensamente degradado nas últimas décadas, apresentando hoje apenas $20 \%$ de sua área original conservada (AGUIAR; CAMARGO, 2004). A degradação desse bioma vem sendo atribuída à expansão agrícola do País, que, segundo Souza et al. (2002), vem ocorrendo de forma irresponsável, sem preocupação com o desperdício do potencial de várias espécies nativas do cerrado. Esse bioma possui uma enorme variedade de espécies arbóreas que poderiam ser aproveitadas racionalmente em diversos setores da economia, além da grande quantidade de espécies que estão sendo destruídas sem que se tenha tido a oportunidade de estudá-las.

Gonçalo-Alves (Astronium fraxinifolium) é uma espécie arbórea de cerrado que, de acordo com a Portaria IBAMA n. 37-N, de 3 de abril de 1992 (IBAMA, 1992), está ameaçada de extinção. Da família Anacardiaceae, é considerada uma espécie pioneira e heliófita, característica de terrenos rochosos e secos, onde forma agrupamentos isolados (LORENZI, 1992) e de maior densidade em áreas de solos com melhores condições químicas (ALMEIDA et al., 1998). Essa espécie possui madeira pesada (densidade de 1,09 $\mathrm{g} \mathrm{cm}^{-3}$ ), compacta, rígida, de grande durabilidade sob condições naturais, o que a classifica como adequada para uso na construção civil e naval, na marcenaria, para confecção de dormentes a portas de fino acabamento, além de ser uma opção para o paisagismo por seu porte médio e beleza da copa (LORENZI, 1992).

Considerando a possibilidade de sua utilização em projetos de reflorestamento e arborização urbana, faz-se necessário produzir mudas que propiciem crescimento satisfatório quando transplantadas para o campo. Para isso, é importante fornecer condições adequadas para o desenvolvimento das mudas, melhorando sua qualidade por meio de nutrição mineral planejada (GONÇALVES et al., 2000) e considerando as informações fornecidas por Duboc e Guerrini (2007) sobre o elevado requerimento nutricional de nitrogênio por essa espécie. Desse modo, a fertilidade do substrato é muito importante, pois deve disponibilizar os nutrientes em quantidades balanceadas, e o conhecimento das exigências nutricionais da espécie é necessário ao estabelecimento de adubação apropriada. Para isso, Mendonça et al. (1999) sugerem a realização de trabalhos que determinem a dosagem de cada nutriente a ser aplicada, dentre os quais o $\mathrm{N}$, que se mostra limitante ao crescimento e à produção florestal (NAMBIAR, 1989).

A atual degradação do cerrado, em acordo com Salati et al. (2006), é um tema de grande importância para o futuro do Brasil, bem como a perda de sua biodiversidade, responsável pelo equilíbrio e pela estabilidade de todo o ecossistema. No cerrado, ainda são encontradas espécies pouco conhecidas, que podem ter potencial econômico e sustentável. Com base nessas premissas é que se desenvolveu este trabalho, com o objetivo de avaliar o efeito de diferentes fontes e doses de N sobre o crescimento de mudas de GonçaloAlves.

\section{MATERIAL E MÉTODOS}

O experimento foi realizado de agosto de 2007 a janeiro de 2008, em estufa, na Faculdade de Engenharia, UNESP - Universidade Estadual Paulista, Campus de Ilha Solteira. O solo (Latossolo Vermelho Distrófico típico) utilizado para a produção das mudas foi passado em peneira com malha de $4 \mathrm{~mm}$, coletado na profundidade de 0,0 a 0,20 m, em cerrado degradado, no município de Selvíria (MS), e apresentou as seguintes características químicas, em análise realizada de acordo com Raij e Quaggio (1983): pH 4,7; 21 g dm³ de matéria orgânica; $12 \mathrm{mg} \mathrm{dm}^{-3}$ de P;1,6 mmolc dm ${ }^{-3}$ de K; 7 mmolc dm $^{-3}$ de Ca; 5 mmolc dm $^{-3}$ de Mg; 29 mmolc dm $^{-3}$ de acidez potencial; 4 mmolc $\mathrm{dm}^{-3} \mathrm{de} \mathrm{Al}$; 13,8 mmolc $\mathrm{dm}^{-3} \mathrm{de}$ soma de bases; CTC de 42,8 mmolc dm ${ }^{-3}$ e 32\% de saturação por bases.

As mudas de Gonçalo-Alves foram produzidas a partir de sementes coletadas em 17 árvores da espécie que germinaram em germobox (20 sementes/germobox). Três dias após a germinação, as plântulas foram acondicionadas individualmente em copos descartáveis (100 mL) contendo o solo descrito, sem nenhum tipo de fertilização. Aos 74 dias, foram transplantadas para sacos plásticos próprios com capacidade para $3 \mathrm{~L}$.

O delineamento experimental utilizado foi o fatorial $3 \times 5$, totalizando 15 tratamentos, com nove repetições. Os tratamentos consistiram de: três fontes de $\mathrm{N}\left(\left(\mathrm{NH}_{4}\right)_{2} \mathrm{SO}_{4}\right.$, $\mathrm{NH}_{4} \mathrm{NO}_{3}$ e $\left.\mathrm{Ca}\left(\mathrm{NO}_{3}\right)_{2}\right)$ e cinco doses de $\mathrm{N}(0 ; 50 ; 100$; 150 e $200 \mathrm{mg} \mathrm{dm}^{-3}$ ). As doses de $\mathrm{N}$ foram aplicadas em cobertura, divididas em quatro aplicações, em intervalos de 30 dias, o que significa que foram aplicados $12,5 \mathrm{mg} \mathrm{dm}^{-3}$ de $\mathrm{N}$ para a dose de $50 ; 25,0 \mathrm{mg} \mathrm{dm}^{-3} \mathrm{de}$ $\mathrm{N}$ para a dose $100 ; 37,5 \mathrm{mg} \mathrm{dm}^{-3}$ de $\mathrm{N}$ para a dose 150

Revista Árvore, Viçosa-MG, v.35, n.3, p.401-411, 2011

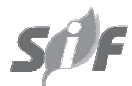


e $50 \mathrm{mg} \mathrm{dm}^{-3}$ para a dose 200 , a cada aplicação feita. Todos os tratamentos receberam $45 \mathrm{mg} \mathrm{dm}^{-3} \mathrm{de}_{2} \mathrm{O}$, 46,8 $\mathrm{mg} \mathrm{dm}^{-3}$ de $\mathrm{P}_{2} \mathrm{O}_{5}, 0,342 \mathrm{~g} \mathrm{dm}^{-3}$ de calcário, incorporados ao solo na forma sólida, como $\mathrm{KCl}$, superfosfato triplo e calcário calcítico, respectivamente, logo após o transplantio das mudas.

Todos os tratamentos foram inoculados com 100 g de solo como reinoculante de microrganismos, coletado na profundidade de 0 a 0,10 m em área de cerrado preservado, próxima do local de coleta do solo do experimento, tratando-se também de um Latossolo Vermelho Distrófico típico.

Medidas de altura $(\mathrm{H})$ das plantas foram tomadas aos 30, 60, 120 e 150 dias após o transplantio para os sacos de mudas que continham o material dos tratamentos. Aos 150 dias de exposição aos tratamentos, quando as plantas tinham 224 dias de idade, o experimento foi desmontado e as mudas avaliadas conforme o diâmetro do coleto (DC), teor de clorofila foliar (TCF), peso da matéria fresca e seca da parte aérea (PMFPAe PMSPA), peso da matéria fresca (PMFR) e seca do sistema radicular (PMSR) e peso da matéria seca total (PMST). Foram obtidas as relações entre altura e diâmetro do coleto (H/DC), altura e peso da matéria seca da parte aérea (H/PMSPA), razão entre peso da matéria seca da parte aérea e do sistema radicular (PMSPA/PMSR) e o índice de qualidade de Dickson (IQD), por meio da fórmula (DICKSON et al., 1960):
$\mathrm{IQD}=$

$\operatorname{PMST}(g)$

H (cm) / DC (mm) + PMSPA (g) / PMSR (g)

O teor de clorofila foliar (TCF) foi medido com um clorofilômetro minolta SPAD - 500, cujas leituras foram feitas em duas folhas adultas do ápice de cada planta, tendo seus valores médios convertidos para $m g \mathrm{~cm}^{-2}$, por meio da equação: $\mathrm{Y}=0,0996 \mathrm{x}-0,152$ (FURLANI JUNIOR et al., 1996).

No final do experimento, o solo passou por nova caracterização química seguindo a metodologia de Raij e Quaggio (1983).

Os dados foram avaliados por meio de análise de variância, com modelo fatorial 3x5, para F até 5\%. As variáveis quantitativas, com respostas significativas, foram comparadas entre si por meio de regressão, e as demais pelo teste de Tukey.

\section{RESULTADOS E DISCUSSÃO}

Os resultados das análises de fertilidade do solo no final do experimento apresentaram diferenças significativas, com exceção de P, Mg e SB (Tabela 1). Para $\mathrm{pH}$, houve diferença estatisticamente significativa para fontes, doses e interação fontes x doses de $\mathrm{N}$ $(F<0,0001)$, foram encontrados os maiores valores de $\mathrm{pH}$ na ausência de $\mathrm{N}$, com redução deste à medida que

Tabela 1 - Médias de fósforo (P-resina), matéria orgânica (MO), potencial de hidrogênio (pH), potássio (K), cálcio (Ca), magnésio (Mg), acidez potencial (H+Al), alumínio (Al), soma de bases (SB), capacidade de troca catiônica (CTC), saturação por bases (V \%), probabilidade de F e coeficiente de variação (CV) para o solo com diferentes fontes e doses de nitrogênio.

Table 1 - Averages of phosphorus (P-resin), organic matter (OM), pH, potassium (K), calcium (Ca), magnesium (Mg), potential acidity $(H+A l)$, aluminum $(A l)$, sum of bases $(S B)$, cation exchange capacity (CTC), base saturation $(V \%), F$ probability and coefficient of variation (CV), for the soil under different sources and doses of nitrogen.

\begin{tabular}{|c|c|c|c|c|c|c|c|c|c|c|c|}
\hline $\begin{array}{l}\text { Fontes de } \\
\text { Variação }\end{array}$ & $\begin{array}{l}\text { P-resina } \\
\text { mg dm }\end{array}$ & $\begin{array}{c}\text { MO } \\
\mathrm{g} \mathrm{dm}^{-3}\end{array}$ & $\begin{array}{c}\mathbf{p H} \\
\mathrm{CaCl}_{2}\end{array}$ & $\mathbf{K}$ & $\mathbf{C a}$ & Mg & $\begin{array}{c}\mathbf{H}+\mathbf{A l} \\
\mathrm{mol}_{\mathrm{c}} \mathrm{dm}\end{array}$ & Al & S B & CTC & $\begin{array}{l}\mathrm{V} \\
\%\end{array}$ \\
\hline $\begin{array}{l}\text { Fontes de } \mathrm{N} \\
\left(\mathrm{NH}_{4}\right)_{2} \mathrm{SO}_{4}\end{array}$ & 3 & $6,0 \mathrm{~b}$ & $4,5 \mathrm{c}$ & 0,80 & $6,13 \mathrm{~b}$ & 5,5 & 24,5 a & 4,3 a & 12,5 & 37,0 a & $34 \mathrm{~b}$ \\
\hline $\mathrm{NH}_{4} \mathrm{NO}_{3}$ & 3 & 6,6 a & $4,6 \mathrm{~b}$ & 0,69 & $5,40 \mathrm{~b}$ & 5,0 & 23,5 a & $3,0 \mathrm{~b}$ & 11,1 & $34,5 \mathrm{ab}$ & $32 \mathrm{~b}$ \\
\hline $\begin{array}{l}\mathrm{Ca}\left(\mathrm{NO}_{3}\right)_{2} \\
\text { Probabilidade de } \mathrm{F}\end{array}$ & 3 & 6,6 a & 4,9 a & 0,73 & 7,73 a & 5,1 & $19,3 \mathrm{~b}$ & $0,4 \mathrm{c}$ & 13,5 & $32,9 \mathrm{~b}$ & 41 a \\
\hline$F^{(1)}$ & . & $4,50^{*}$ & $73,93^{* *}$ & $1,23^{\mathrm{ns}}$ & $8,28^{* *}$ & 0,74 & $76,00^{* *}$ & $96,46^{* *}$ & $2,74^{\mathrm{ns}}$ & $6,71^{* *}$ & $15,05^{* *}$ \\
\hline $\mathrm{D}^{(2)}$ & . & $1,33^{\text {ns }}$ & $26,91^{* *}$ & $14,37^{* *}$ & $2,64^{\mathrm{ns}}$ & 1,60 & $51,49^{* *}$ & $44,95^{* *}$ & $1,45^{\mathrm{ns}}$ & $11,45^{* *}$ & $2,51^{\mathrm{ns}}$ \\
\hline F x D & . & $2,21^{\mathrm{ns}}$ & $8,53^{* *}$ & $0,96^{\mathrm{ns}}$ & $1,44^{\mathrm{ns}}$ & 0,76 & $11,88^{* *}$ & $14,95^{* *}$ & $0,84^{\mathrm{ns}}$ & $1,67^{\mathrm{ns}}$ & $2,13^{\mathrm{ns}}$ \\
\hline CV (\%) & . & 10 & 2 & 27 & 25 & 25 & 5 & 31 & 23 & 9 & 13 \\
\hline
\end{tabular}

Médias seguidas de mesma letra nas colunas, por fonte de variação, não diferem significativamente entre si pelo teste de Tukey a 5\%. ns=valores não significativos; ${ }^{* *} \mathrm{e}^{*}=$ valores significativos para $\mathrm{P}<0,01 \mathrm{e}<0,05$, respectivamente

(1) $\mathrm{F}=$ Fontes de $\mathrm{N}$.

(2) $\mathrm{D}=$ Doses de $\mathrm{N}$. 
as doses de $\mathrm{N}$ aumentaram (Figura 1a). Isso pode ser explicado pela acidificação do solo promovida por alguns fertilizantes nitrogenados, que produzem $\mathrm{H}^{+}$, durante reações de nitrificação (TEIXEIRA et al., 2001). Entre as fontes de $\mathrm{N}$ testadas, o $\mathrm{Ca}\left(\mathrm{NO}_{3}\right)_{2}$ promoveu incrementos no $\mathrm{pH}$, significativos em relação às demais fontes $\left(\left(\mathrm{NH}_{4}\right)_{2} \mathrm{SO}_{4}, \mathrm{NH}_{4} \mathrm{NO}_{3}\right)$, a partir da dose de 100 $\mathrm{mg} \mathrm{dm}^{-3}$ de $\mathrm{N}$ (Tabela 2). As demais fontes promoveram igualmente uma acidificação significativa a partir da dose citada (100 $\mathrm{mg} \mathrm{dm}^{-3}$ ). Neto et al. (2006), ao confirmar a redução do $\mathrm{pH}$ do solo como efeito da nitrificação na utilização de $\left(\mathrm{NH}_{4}\right)_{2} \mathrm{SO}_{4}$, em experimento realizado com mamoeiro, corroboram esses resultados.

(a)

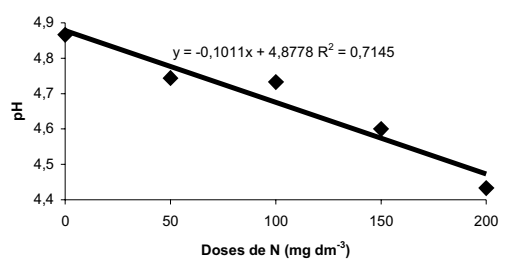

(c)

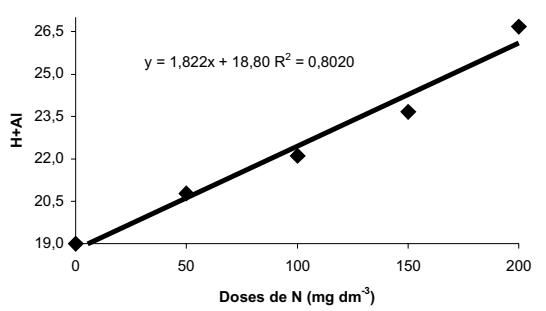

A aplicação de $\mathrm{Ca}\left(\mathrm{NO}_{3}\right)_{2}$ causou, além de incrementos nos valores do $\mathrm{pH}$, aumentos significativos de cálcio, redução da acidez potencial $(\mathrm{H}+\mathrm{Al})$ e do Al do solo, permitindo afirmar que, das três fontes de $\mathrm{N}$ utilizadas, o $\mathrm{Ca}\left(\mathrm{NO}_{3}\right)_{2}$ apresentou resultados um pouco melhores (Tabela 1). As equações de regressão obtidas para as variáveis que apresentaram respostas significativas para doses de $\mathrm{N}$ (Tabela 1 ) seguem apresentadas na Figura 1, onde se observa comportamento linear decrescente em relação ao incremento nas doses de $\mathrm{N}$ para $\mathrm{pH}$, e comportamento oposto, isto é, linear crescente para acidez potencial $(\mathrm{H}+\mathrm{Al})$, alumínio $(\mathrm{Al})$ e CTC, comportamento inverso

(b)

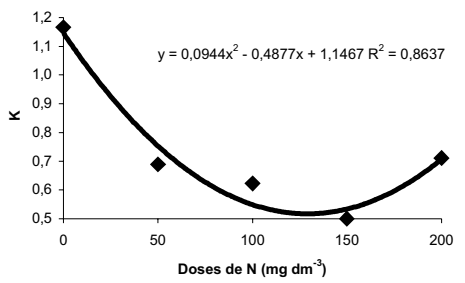

(d)

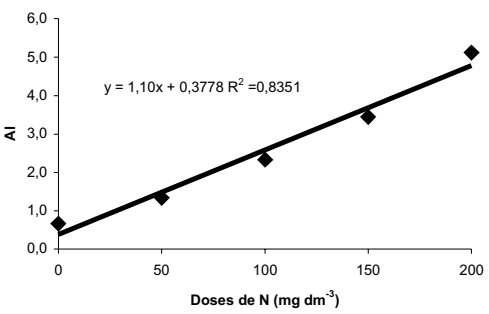

(e)

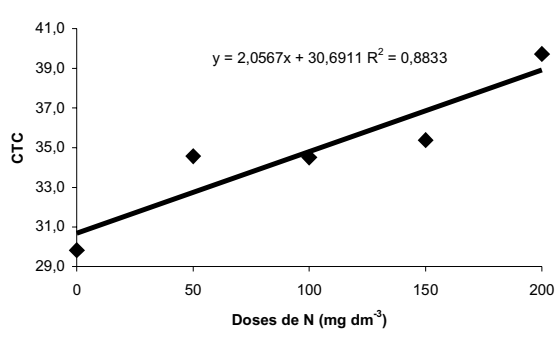

Figura 1- Equações de regressão ajustadas para (a) potencial de hidrogênio (pH), (b) potássio (K), (c) acidez potencial (H+Al), (d) alumínio (Al) e (e) capacidade de troca catiônica (CTC) para o solo, em função das doses de nitrogênio aplicadas (significância e valores de t para as equações no Anexo 1).

Figure 1 - Regression equations adjusted to (a) pH, (b) potassium (K), (c) potential acidity (H+Al), (d) aluminum (Al), and (e) cation exchange capacity (CTC), under the effect of different levels of nitrogen (significance and $t$ values to equations in annex1).

Revista Árvore, Viçosa-MG, v.35, n.3, p.401-411, 2011 
Tabela 2 - Desdobramento da interação fontes x doses de Nitrogênio para potencial de hidrogênio (pH), acidez potencial $(\mathrm{H}+\mathrm{Al})$ e alumínio $(\mathrm{Al})$.

Table 2 - Interaction sources $\times$ levels of Nitrogen for $p H$, potential acidity $(H+A l)$, and aluminum $(A l)$.

\begin{tabular}{|c|c|c|c|c|c|c|c|c|c|c|}
\hline \multirow{2}{*}{$\begin{array}{l}\text { Fontes } \\
\text { de } \mathrm{N}\end{array}$} & \multicolumn{8}{|c|}{ Doses de $\mathrm{N}$ em $\mathrm{mg.dm}^{-3}$} & \multirow[t]{2}{*}{ Equações* } & \multirow[t]{2}{*}{$\mathrm{R}^{2}$} \\
\hline & 0 & & 50 & & 100 & 150 & 200 & & & \\
\hline$\left(\mathrm{NH}_{4}\right)_{2} \mathrm{SO}_{4}$ & 4,87 & A & 4,60 & A & $4,53 B$ & $\begin{array}{c}\mathrm{pH} \\
4,33 \mathrm{~B}\end{array}$ & 4,20 & B & $\begin{array}{l}Y=-0,1600^{* *} \mathrm{x}+4,8267^{* *} \\
\mathrm{t}-15,54 \quad 191,41\end{array}$ & 0,9489 \\
\hline $\mathrm{NH}_{4} \mathrm{NO}_{3}$ & 4,87 & A & 4,80 & A & $4,57 \mathrm{~B}$ & $4,50 \mathrm{~B}$ & 4,30 & B & $\begin{array}{l}Y=-0,1433^{* *} x+4,8933^{* *} \\
t \quad-6,44 \quad 89,80\end{array}$ & 0,7615 \\
\hline $\mathrm{Ca}\left(\mathrm{NO}_{3}\right)_{2}$ & 4,87 & A & 4,83 & A & $5,10 \mathrm{~A}$ & $4,97 \mathrm{~A}$ & 4,80 & A & ns & - \\
\hline$\left(\mathrm{NH}_{4}\right)_{2} \mathrm{SO}_{4}$ & 19,0 & A & 21,7 & A & $24,0 \mathrm{~B}$ & $\begin{array}{l}\mathrm{H}+\mathrm{Al} \\
26,7 \mathrm{~B}\end{array}$ & 31,3 & B & $\begin{array}{l}Y=2,9667^{* *} \mathrm{X}+18,6000^{* *} \\
\mathrm{t} 10,75\end{array}$ & 0,8989 \\
\hline $\mathrm{NH}_{4} \mathrm{NO}_{3}$ & 19,0 & A & 21,0 & A & $23,3 \mathrm{~B}$ & $25,0 \mathrm{~B}$ & 29,0 & B & $\begin{array}{l}Y=2,4000^{* *} \mathrm{X}+18,6667^{* *} \\
\mathrm{t} \quad 10,36 \quad 32,89\end{array}$ & 0,8919 \\
\hline $\mathrm{Ca}\left(\mathrm{NO}_{3}\right)_{2}$ & 19,0 & A & 19,7 & A & $19,0 \mathrm{~A}$ & $19,3 \mathrm{~A}$ & 19,7 & A & ns & - \\
\hline$\left(\mathrm{NH}_{4}\right)_{2} \mathrm{SO}_{4}$ & 0,7 & A & 2,0 & A & $4,0 \mathrm{~B}$ & $\begin{array}{cc}\mathrm{Al} \\
6,0 \mathrm{~B}\end{array}$ & 9,0 & $\mathrm{C}$ & 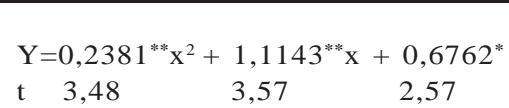 & 0,9789 \\
\hline $\mathrm{NH}_{4} \mathrm{NO}_{3}$ & 0,7 & A & 1,3 & A & $3,0 \mathrm{~B}$ & $4,0 \mathrm{~B}$ & 6,0 & B & $\begin{array}{ll}Y=1,3333^{* *} \mathrm{X} & +0,3333^{\mathrm{ns}} \\
\mathrm{t} \quad 6,88 & 0,70\end{array}$ & 0,7843 \\
\hline $\mathrm{Ca}\left(\mathrm{NO}_{3}\right)_{2}$ & 0,7 & A & 0,7 & A & $0,0 \mathrm{~A}$ & $0,3 \mathrm{~A}$ & 0,3 & A & ns & -- \\
\hline
\end{tabular}

Médias seguidas de mesma letra nas colunas, por variável e por dose de N, não diferem significativamente entre si pelo teste de Tukey a $5 \%$.

* Equações de Regressão para doses, dentro de cada uma das fontes, sua significância e valor de t. ns = não significativo.

ao do pH, o que está de acordo com Mello et al. (1983), pois, à medida que o $\mathrm{pH}$ diminui, ocorrem incrementos em Al trocável e $\mathrm{H}^{+}$, com efeitos sobre a CTC do solo. O potássio apresenta comportamento quadrático negativo, isto é, perda de K com incrementos nas doses de N, o que Luchese et al. (2001) explicam como uma característica do processo de acidificação dos solos.

Em relação ao solo em sua condição inicial, verifica-se redução da matéria orgânica de 21 para $6 \mathrm{mg} \mathrm{dm}^{-3}$, o que pode ser explicado pelas condições em que o experimento foi conduzido, isto é, temperaturas elevadas (estufa) e água em abundância, via rega, o que pode ter contribuído para sua redução acentuada (MIELNICZUK et al., 2003).

As medidas de altura de Gonçalo-Alves, tomadas ao longo do tempo, foram influenciadas pelas doses de $\mathrm{N}$, pelo tempo e pela interação desses fatores (Tabela 3), e apresentaram-se semelhantes em todas as doses de N entre 30 e 60 dias de exposição aos tratamentos (Figura 2). Próximo dos 120 dias, verifica-se intensificação no crescimento das mudas (Tabela 4), devido ao estádio de desenvolvimento das plantas e às doses de $\mathrm{N}$ aplicadas, o que, aos 150 dias, aponta para a dose de
72,5 $\mathrm{mg} \mathrm{dm}^{-3}$ de $\mathrm{N}$ como suficiente para o crescimento da planta em altura, que apresenta comportamento quadrático (Figura 3a), semelhante ao verificado por Marques et al. (2006b), em experimento realizado com mudas de jacarandá-da-bahia.

Nenhum dos parâmetros considerados para avaliação das mudas foi influenciado pelas fontes de $\mathrm{N}$ utilizadas (Tabela 5). Aaltura, o diâmetro do coleto, o teor de clorofila foliar, o peso da matéria fresca e seca da parte aérea, o peso da matéria fresca e seca do sistema radicular, a razão peso da matéria seca da parte aérea/peso da matéria seca da raiz, o peso seco total e os valores do Índice de Qualidade de Dickson apresentaram diferenças significativas apenas para as doses de $\mathrm{N}$ testadas (Tabela 5). Marques et al. (2006a) também verificaram ausência de resposta positiva para altura, diâmetro do coleto, peso da matéria seca total e peso da matéria seca da raiz de mudas de sabiá para diferentes fontes de N, encontrando respostas às diferentes doses de $\mathrm{N}$ aplicadas. Duboc e Guerrini (2007) também encontraram resultados positivos para diâmetro do colo de plantas de Gonçalo-Alves, em experimento conduzido em campo em função das doses de $\mathrm{N}$ aplicadas, que variaram de 0 a $40 \mathrm{~kg} \mathrm{ha}^{-1}$.

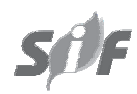

Revista Árvore, Viçosa-MG, v.35, n.3, p.401-411, 2011 
Tabela 3 - Médias de altura das mudas de Gonçalo-Alves em função de diferentes fontes e doses de nitrogênio ao longo do tempo. Probabilidade de F e Coeficiente de Variação (CV).

Table 3 - Means of heights of Gonçalo Alves seedlings as a function of different sources and doses of nitrogen, over time, F Probability and Coefficient of Variation ( $\mathrm{CV}$ ).

\begin{tabular}{lc}
\hline Fontes de Variação & ALTURA (cm) \\
\hline Fontes de N (F) & 12,1 \\
$\left(\mathrm{NH}_{4}\right)_{2} \mathrm{SO}_{4}$ & 12,3 \\
$\mathrm{NH}_{4} \mathrm{NO}_{3}$ & 11,7 \\
$\mathrm{Ca}\left(\mathrm{NO}_{3}\right)_{2}$ & $1,63^{\mathrm{ns}}$ \\
Probabilidade de F & $44,70^{* *}$ \\
$\mathrm{~F}^{(1)}$ & $593,69^{* * *}$ \\
$D^{(2)}$ & $1,72^{\text {ns }}$ \\
$T^{(3)}$ & $0,29^{\mathrm{ns}}$ \\
$\mathrm{FxD}$ & $12,69^{* *}$ \\
$\mathrm{FxT}$ & $1,00^{\mathrm{ns}}$ \\
TxD & 20 \\
FxDxT & 20 \\
$\mathrm{CV}(\%)$ &
\end{tabular}

(1) $\mathrm{F}=$ Fontes de Nitrogênio; (2) $\mathrm{D}=$ Doses de Nitrogênio; e ${ }^{(3)} \mathrm{T}=$ Tempo de experimentação (150 dias).

Médias seguidas de mesma letra nas colunas, por fonte de variação, não diferem significativamente entre si pelo teste de Tukey a 5\%. ${ }^{\mathrm{ns}}=$ valores não significativos; ${ }^{* * *} \mathrm{e}^{*}=$ valores significativos para $\mathrm{P}<0,01 \mathrm{e}<0,05$, respectivamente.

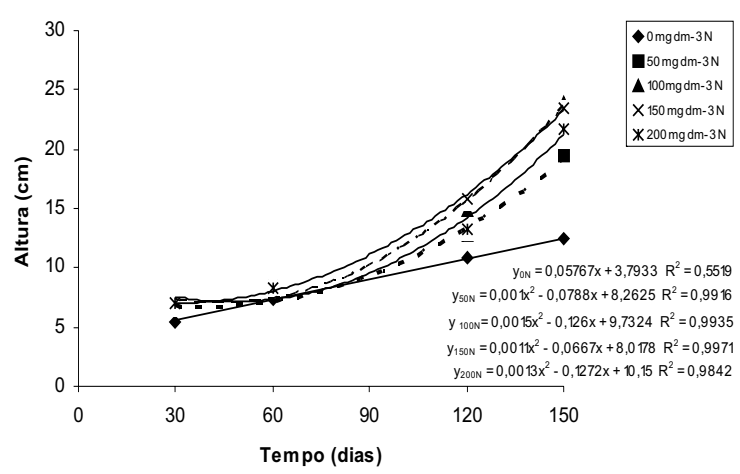

Figura 2 - Equações de regressão ajustadas para altura das mudas de Gonçalo-Alves, ao longo do tempo de exposição das mudas aos tratamentos e sob o efeito de diferentes doses de nitrogênio (significância e valores de t para as equações no Anexo 1).

Figure 2-Regression equations adjusted to height of GonçaloAlves seedlings, over time in experimental conditions, under the effect of different doses of nitrogen (significance and t values to equations at annex 1).

O diâmetro de coleto máximo ocorreu com a dose de 65,75 $\mathrm{mg} \mathrm{dm}^{-3}$ (Figura 3b), com comportamento quadrático, em relação às doses de $\mathrm{N}$ aplicadas. $\mathrm{O}$ teor de clorofila foliar apresentou valor máximo para a dose de 68,75 $\mathrm{mg} \mathrm{dm}^{-3}$ de N (Figura 3c), e o menor valor na ausência de $\mathrm{N}$, o que justifica, segundo Argenta et al. (2001), a aplicação de N, pois esse é encontrado em várias enzimas constituintes dos cloroplastos, portanto essencial ao desenvolvimento da planta.
Os pesos da matéria fresca e seca da parte aérea e da matéria fresca e seca da raiz apresentaram comportamento quadrático em relação às doses de $\mathrm{N}$ aplicadas, com valores máximos com as doses de 64,25; 63,75; 60,50; e $61,75 \mathrm{mg} \mathrm{dm}^{-3}$ de $\mathrm{N}$, respectivamente, (Figuras 3d, 3e, 3f e 4a), não tendo sido observados melhores resultados na dose mais elevada de $\mathrm{N}$ (200 $\mathrm{mg} \mathrm{dm}^{-3}$ ), permitindo indicar a dose de 64,25 $\mathrm{mg} \mathrm{dm}^{-3}$ como suficiente para o crescimento satisfatório de mudas de Gonçalo-Alves para esses parâmetros.

Esses resultados são corroborados pelo índice de qualidade de Dickson, que indica a qualidade da muda produzida e que quanto maior o valor obtido melhor será a qualidade da muda (GOMES, 2001), cujo valor máximo foi encontrado para a dose de $59 \mathrm{mg} \mathrm{dm}^{-3}$, segundo equação quadrática que representa este comportamento (Figura 4b).

Nota-se ausência de efeitos tanto para fonte como para as doses de $\mathrm{N}$ aplicadas sobre as relações H/DC e H/PMSPA (Tabela 5). No entanto, a razão entre o peso da matéria seca da parte aérea e o peso da matéria seca da raiz (PMSPA/PMSR), bem como o peso da matéria seca total, apresentaram diferenças significativas para as doses de $\mathrm{N}$ aplicadas (Tabela 5), tendo na ausência de $\mathrm{N}$ os menores valores (Figura 4c e 4d), isto é, menor produção de biomassa. Segundo Parviainen (1981 apud MARQUES et al., 2006a), a razão PMSPA/PMSR pode 
Tabela 4 - Desdobramento da interação tempo x doses de Nitrogênio para altura das mudas de Gonçalo-Alves.

Table 4 - Interaction time $\times$ levels of Nitrogen for height of Gonçalo Alves seedlings.

\begin{tabular}{|c|c|c|}
\hline $\begin{array}{l}\text { Tempo de Experimentação (dias) } \\
\text { para as doses de } \mathrm{N} \text { aplicadas }\end{array}$ & Equações & $\mathrm{R}^{2}$ \\
\hline 30 & $\begin{array}{ccc}Y=-0,1437^{*} x^{2 * *}+1,2108^{* *} \mathrm{x}+4,5067 \\
\mathrm{t}=-2,51 & 3,46 & 9,80\end{array}$ & 0,6737 \\
\hline 60 & $\begin{array}{ll}Y=0,2694^{* *} \mathrm{x}+ & 7,1772^{* *} \\
\mathrm{t}=3,75 & 30,09\end{array}$ & 0,7376 \\
\hline 120 & $\begin{array}{ll}Y=0,8006^{* *} x+ & 11,0728^{* *} \\
t=3,34 & 13,91\end{array}$ & 0,8123 \\
\hline 150 & $\begin{array}{ll}Y=2,2744^{* *} \mathrm{X}+ & 13,3718^{* *} \\
\mathrm{t}=6,81 & 12,07\end{array}$ & 0,7450 \\
\hline $\begin{array}{l}\text { Doses de } \mathrm{N}\left(\mathrm{mg} \mathrm{dm}^{-3}\right) \\
\text { para os tempos analisados }\end{array}$ & Equações & $\mathrm{R}^{2}$ \\
\hline 0 & $\begin{array}{l}\mathrm{Y}=2,4200^{* *} \mathrm{X}+2,9303^{* *} \\
\mathrm{t}=9,06 \quad 4,01\end{array}$ & 0,7400 \\
\hline 50 & $\begin{array}{l}Y=1,3528^{* *} x^{2}-2,4061^{*} x+7,4667^{* *} \\
t=6,11 \\
t=2,14\end{array}$ & 0,8832 \\
\hline 100 & $\begin{array}{ccc}Y=8,9319^{* *}- & 4,1697^{* *} \mathrm{x}+1,9958^{* *} \mathrm{x}^{2} \\
\mathrm{t}=6,14 & -3,14 & 7,64\end{array}$ & 0,9074 \\
\hline 150 & $\begin{array}{llc}Y= & 1,5167^{* *} x^{2}- & 1,8478^{n s} x+6,8883^{* *} \\
t=6,56 & -1,57 & 5,35\end{array}$ & 0,9217 \\
\hline 200 & $\begin{array}{lll}Y=1,8097^{* *} x^{2}- & 4,1636^{*} x+9,4153^{* *} \\
t=4,68 & -2,12 & 4,37\end{array}$ & 0,7622 \\
\hline
\end{tabular}

* Equações de Regressão para altura, nos diferentes tempos e doses de Nitrogênio, sua significância e valor de t. ns = não significativo.

ser eficiente para expressar a qualidade das mudas, pois indica a proporção de crescimento entre parte aérea e raiz, permitindo inferir que as mudas de GonçaloAlves de melhor qualidade são aquelas que apresentaram maior valor para as variáveis citadas, o que ocorreu para PMSPA/PMSR em comportamento quadrático, com valor de máximo (Figura 4c) para a dose de $68,50 \mathrm{mg} \mathrm{dm}^{-3}$ de $\mathrm{N}$ e com $62,00 \mathrm{mg} \mathrm{dm}^{-3}$ de $\mathrm{N}$ para peso da matéria seca total (Figura 4d).

Pode-se dizer que a razão PMSPA/PMSR e o peso da matéria seca total foram influenciados positivamente pela aplicação de N, isto é, seus valores aumentaram com a aplicação de $\mathrm{N}$, até o ponto de máximo citado. De acordo com Azevedo (2003 apud BERNARDINO et al., 2005), a produção de matéria seca tem sido considerada um dos melhores parâmetros para caracterizar a qualidade de mudas.

Todos os parâmetros de planta avaliados com comportamento significativo (Tabela 5) apresentaram na regressão comportamento quadrático (Figuras 3 e 4), indicando que, para as condições do experimento, a dose de 72,50 $\mathrm{mg} \mathrm{dm}^{-3}$, considerando os pontos máximos calculados, seria suficiente para promover resultados satisfatórios para a maioria das variáveis analisadas para planta.

As equações de regressão apresentadas têm seus valores de T e significância dos termos apresentados no Anexo 1.

\section{CONCLUSÕES}

O crescimento das mudas de Gonçalo-Alves não foi influenciado pelas fontes de $\mathrm{N}\left(\left(\mathrm{NH}_{4}\right)_{2} \mathrm{SO}_{4}, \mathrm{NH}_{4} \mathrm{NO}_{3} \mathrm{e}\right.$ $\left.\mathrm{Ca}\left(\mathrm{NO}_{3}\right)_{2}\right)$ utilizadas, mas responderam às doses de $\mathrm{N}$ aplicadas.

A altura da planta, o diâmetro do coleto, o teor de clorofila foliar, o peso da matéria fresca e seca da parte aérea e do sistema radicular, o peso seco total, bem como o IQD e a razão entre o peso da matéria seca da parte aérea e do sistema radicular, apresentaram seus valores máximos com doses entre 59,0 e 72,5 $\mathrm{mg} \mathrm{dm}^{-3} \mathrm{de} \mathrm{N}$.

As análises realizadas indicam a dose de $72,5 \mathrm{mg} \mathrm{dm}^{-3}$ de $\mathrm{N}$ como suficiente para o crescimento satisfatório dessas mudas.

Revista Árvore, Viçosa-MG, v.35, n.3, p.401-411, 2011 
(a)

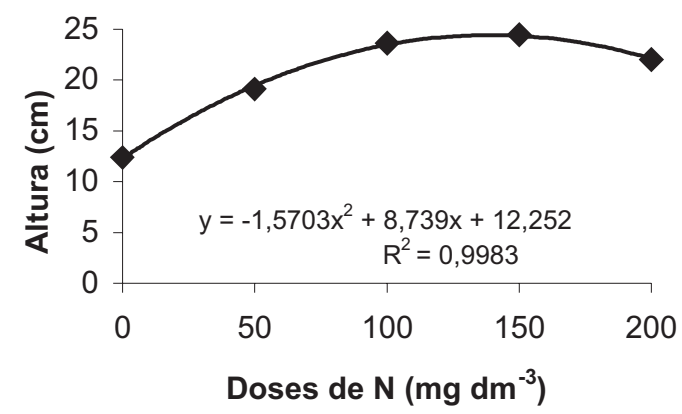

(c)

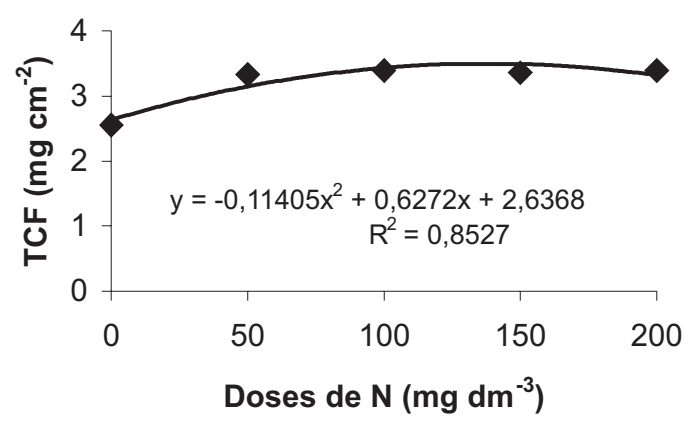

(e)

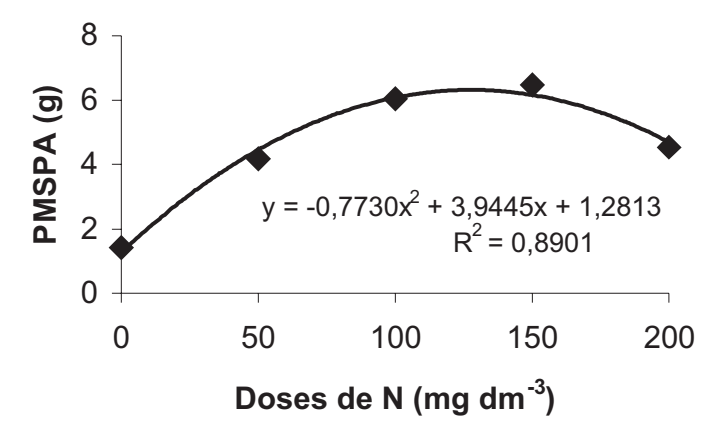

(b)

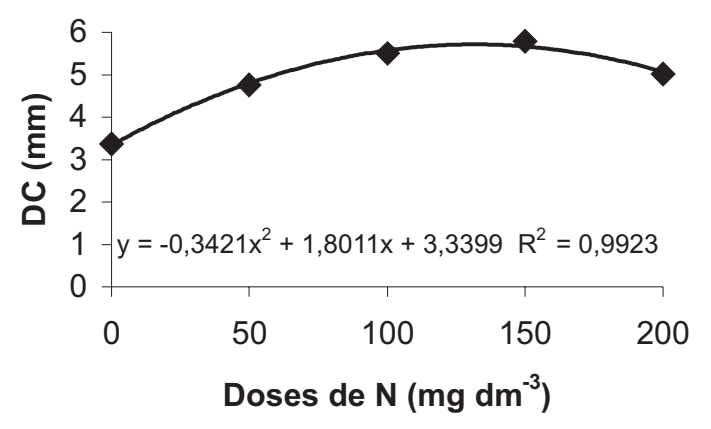

(d)

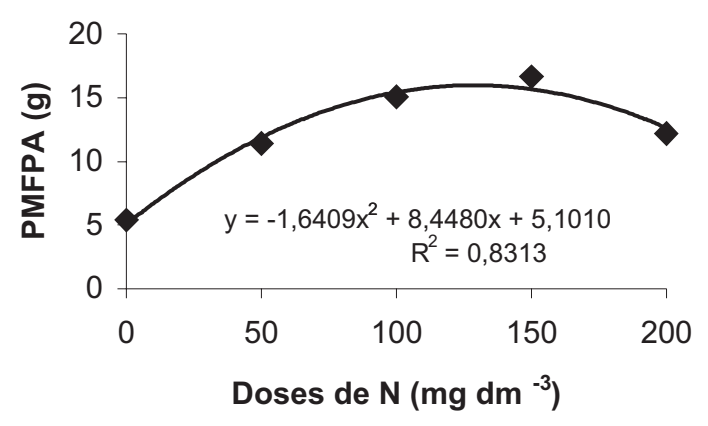

(f)

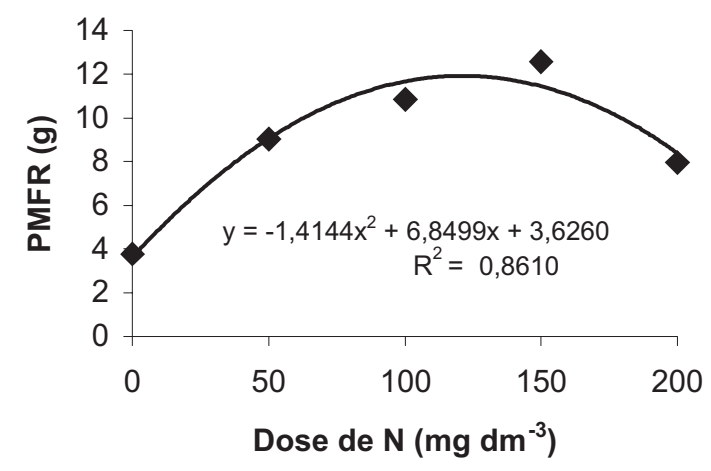

Figura 3 - (a) Altura; (b) diâmetro do coleto (CD); (c) teor de clorofila foliar (TCT); (d) peso da matéria fresca da parte aérea (PMFPA); (e) peso da matéria seca da parte aérea (PMSPA); e (f) peso da matéria fresca da raiz (PMFR) do Gonçalo-Alves, aos 150 dias de cultivo nos tratamentos, em resposta às doses de $\mathrm{N}$ aplicadas (significância e valores de t para as equações no anexo 1$)$.

Figure 3 - Gonçalo-Alves (a) height; (b) stem diameter (DC); (c) leaf chloroplyll content (TCT); (d) shoot fresh weight (PMFPA); (e) shot dry weight (PMSPA); and ( $f$ root fresh matter weight (PMFR), at 150 days under treatments, in response to applied nitrogen levels (significance and t values to equations at annex 1 ).

Revista Árvore, Viçosa-MG, v.35, n.3, p.401-411, 2011 
Tabela 5 - Médias de altura (H), diâmetro do coleto (DC), teor de clorofila foliar (TCF), peso da matéria fresca (PMSPA) e seca da parte aérea (PMSPA) e do sistema radicular (PMFR e PMSR), índice de qualidade de Dickson (IQD), relação altura/diâmetro do coleto (H/DC), altura/peso da matéria seca da parte aérea (H/PMSPA), peso da matéria seca da parte aérea/peso da matéria seca da raiz (PMSPA/PMSR), peso da matéria seca total (PMST), probabilidade de F e coeficiente de variação experimental (CV), de mudas de Gonçalo-Alves em diferentes fontes e doses de nitrogênio, aos 150 dias de cultivo nos tratamentos.

Table 5 - Plant height (H), stem diameter (DC), leaf chlorophyll content (TCF), weight of fresh matter (PMSPA) and dry matter of shoot (PMSPA) and root system (PMFR and PMSR), Dickson quality index (IQD), relation height/stem diameter (H/DC), height/aerial part dry matter weight (H/PMSPA), root:shoot dry weight ratio (PMSPA/PMSR), total dry matter weight (PMST), F probability and experimental coefficient of variation (CV), of Gonçalo-Alves seedlings under different sources and doses of nitrogen, at 150 days under treatments.

\begin{tabular}{|c|c|c|c|c|c|c|c|c|c|c|c|c|}
\hline $\begin{array}{l}\text { Fontes de } \\
\text { Variação }\end{array}$ & $\begin{array}{c}\mathbf{H} \\
(\mathbf{c m})\end{array}$ & $\begin{array}{c}\text { DC } \\
(\mathbf{m m})\end{array}$ & $\begin{array}{c}\text { TCF } \\
\mathrm{mg} \mathrm{cm}^{-2}\end{array}$ & $\begin{array}{l}\text { PMFPA } \\
\text { ') (g) }\end{array}$ & $\begin{array}{c}\text { PMSPA } \\
\text { (g) }\end{array}$ & $\begin{array}{c}\text { PMFR } \\
\text { (g) }\end{array}$ & $\begin{array}{c}\text { PMSR } \\
\text { (g) }\end{array}$ & H/DC & H/PMSPA & PMSPA/PMSR & PMST & IQD \\
\hline $\begin{array}{l}\text { Fontes de I } \\
\left(\mathrm{NH}_{4}\right)_{2} \mathrm{SO}_{4}\end{array}$ & $\begin{array}{l}\mathbf{N}(\mathbf{F}) \\
20,64\end{array}$ & 4,85 & 3,26 & 12,91 & 4,31 & 9,56 & 2,70 & 4,26 & 4,79 & 1,63 & 7,01 & 1,21 \\
\hline $\mathrm{NH}_{4} \mathrm{NO}_{3}$ & 20,41 & 5,16 & 3,19 & 12,63 & 4,83 & 8,93 & 3,14 & 3,96 & 3 & & 7,97 & 1,45 \\
\hline $\mathrm{Ca}\left(\mathrm{NO}_{3}^{3}\right)_{2}$ & 19,88 & 4,66 & 3,18 & 10,92 & 4,45 & 8,02 & 2,74 & 4,26 & 4,47 & 1,62 & 7,19 & 1,21 \\
\hline $\begin{array}{l}\text { Probabilid } \\
\mathrm{F}^{(1)}\end{array}$ & $\begin{array}{c}\text { ide de F } \\
0,09^{\text {ns }}\end{array}$ & $0,75^{\mathrm{ns}}$ & $0,20^{\mathrm{ns}}$ & $0,67^{\mathrm{ns}}$ & $0,25^{\text {ns }}$ & $0,71^{\mathrm{ns}}$ & $0,46^{\mathrm{ns}}$ & $1,79^{\text {ns }}$ & & & $0,44^{\mathrm{ns}}$ & $0,78^{\text {ns }}$ \\
\hline $\mathrm{D}^{(2)}$ & $8,45^{* *}$ & $6,46^{* *}$ & $9,12^{* *}$ & $6,52^{* *}$ & $8,11^{* *}$ & $7,86^{* *}$ & $6,55^{* *}$ & $1,65^{\text {ns }}$ & & & $9,32^{* *}$ & $6,65^{* *}$ \\
\hline & $0,30^{\mathrm{ns}}$ & $0,27^{\text {ns }}$ & $0,54^{\mathrm{ns}}$ & $0,22^{\mathrm{ns}}$ & $0,29^{\text {ns }}$ & $0,26^{\mathrm{ns}}$ & $0,43^{\text {ns }}$ & $1,21^{\mathrm{ns}}$ & $1,22^{\mathrm{ns}}$ & $0,48^{\text {ns }}$ & $0,31^{\mathrm{ns}}$ & $0,56^{\text {ns }}$ \\
\hline CV (\%) & 25 & 23 & 11 & 21 & 23 & 19 & 20 & 13 & 12 & 16 & 21 & 23 \\
\hline
\end{tabular}

${ }^{(1)} \mathrm{F}=$ Fontes de Nitrogênio; e ${ }^{(2)} \mathrm{D}=$ Doses de Nitrogênio.

Médias seguidas de mesma letra, nas colunas, por fonte de variação, não diferem significativamente entre si pelo teste de Tukey a 5\%. ${ }^{n s}=$ valores não significativos; ${ }^{* *} \mathrm{e}^{*}=$ valores significativos para $\mathrm{P}<0,01 \mathrm{e}<0,05$, respectivamente.

(a)

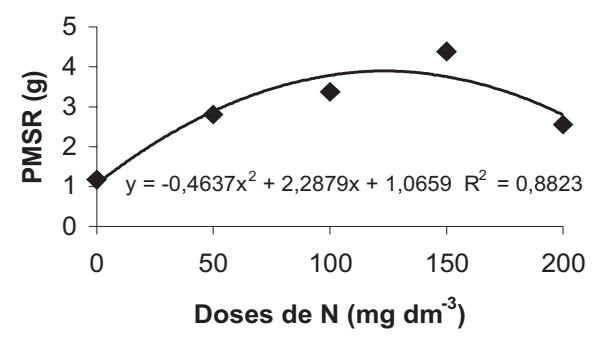

(c)

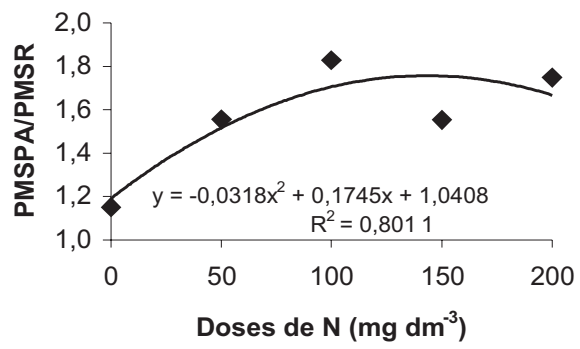

(b)

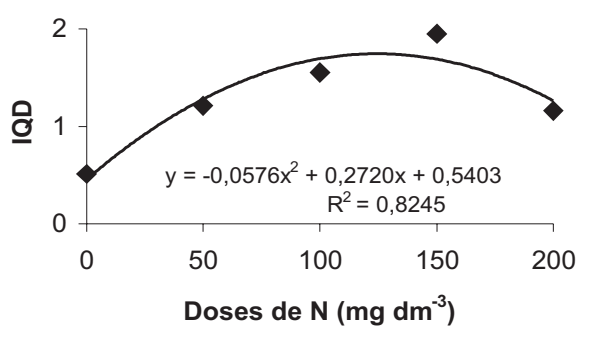

(d)

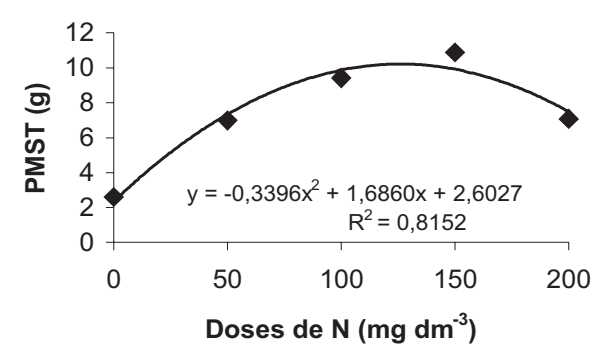

Figura 4 - (a) Peso da matéria seca da raiz (PMSR); (b) índice de qualidade de Dikson (IQD); (c) relação matéria seca da parte aérea/matéria seca da raiz (PMSPA/PMSR); e (d) peso da matéria seca total (PMST) do Gonçalo-Alves, em resposta às doses de $\mathrm{N}$ aplicadas (significância e valores de t para as equações no anexo 1).

Figure 4-Gonçalo-Alves (a) root dry matter weight (PMSR); (b) Dikson quality index (IQD); (c) root:shoot dry weight ratio (PMSPA/PMSR); (d) total dry matter weight (PMST) in response to applied nitrogen levels (significance and $t$ values to equations at annex 1$)$. 
Anexo 1- Equações de regressão, significância de seus termos e respectivos valores de t.

Annex 1 - Regression equations, significance of its terms and their values of $t$.

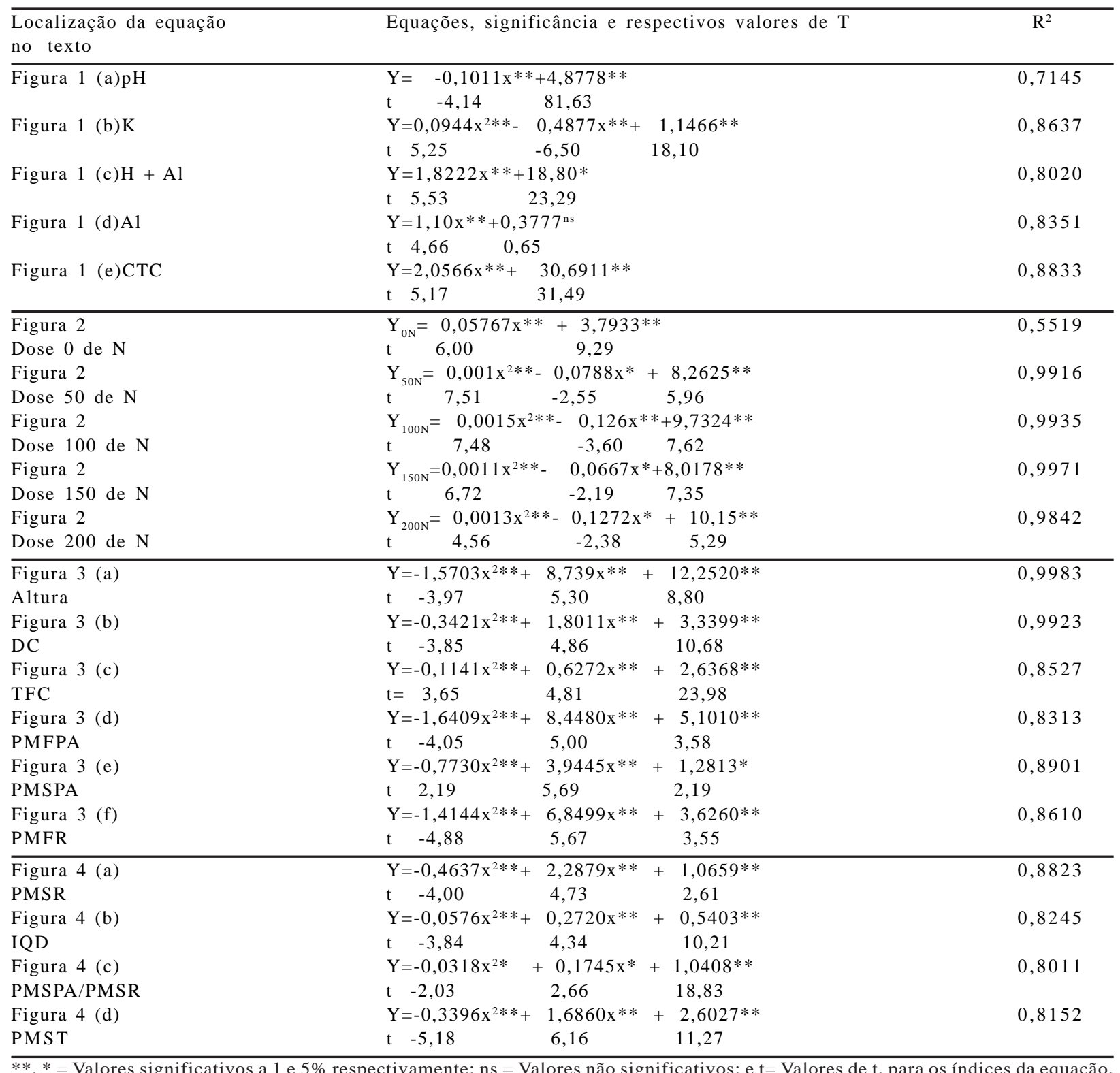
para $\mathrm{P}<0,05$

\section{REFERÊNCIAS}

AGUiAR, L. M. S.; CAMARGO, A. (Orgs.).

Ecologia e caracterização do

cerrado. Brasília: Embrapa, Informação

Tecnológica, 2004. 249p.
ALMEIDA, S. P. et al. Cerrado: espécies vegetais úteis. Planaltina: Embrapa-CPAC, 1998. p.67-71.

ARGENTA, G.; SILVA, P. R. F.; BORTOLINI, C. G. Clorofila na folha como indicador do nível de nitrogênio em cereais. Ciência Rural, v. 31, n. 4, p.715-722, 2001.

Revista Árvore, Viçosa-MG, v.35, n.3, p.401-411, 2011 
BERNARDINO, D. C. S. et al. Crescimento e qualidade de mudas de Anadenanthera macrocarpa (BENTH.) Brenan em resposta à saturação por bases do substrato. Revista Árvore, v. 29, n. 6, p. 863-870, 2005

CARDOSO NETO, F.; GUERRA, H. O. C.; CHAVES, L. H. G. Nitrogênio residual em solos adubados. Caatinga, v. 19, n. 2, p.161-168, 2006.

DICKSON, A.; LEAF, A. L.; HOSNER, J. F. Quality appraisal of white spruce and white pine seedling stock in nurseries. Forestry Chronicle, v. 36, p.10-13, 1960.

DUBOC, E.; GUERRINI, I. A. Crescimento inicial e sobrevivência de espécies florestais de matas de galeria no domínio do cerrado em resposta à fertilização. Energia Agricola, v. 22, n. 1, p. 42-60, 2007.

FURLANI JUNIOR, E. et al. Correlação entre leituras de clorofila e níveis de nitrogênio aplicados em feijoeiro. Bragantia, v. 55, n. 1, p. 171-175, 1996.

GOMES, J. M. Parâmetros morfológicos na avaliação da qualidade de mudas de Eucalyptus grandis, produzidas em diferentes tamanhos de tubete e de dosagens de N-P-K. 2001. 126f. Tese (Doutorado em Ciência Florestal) - Universidade Federal de Viçosa, Viçosa, MG, 2001.

GONÇALVES, J. L. M. et al. Produção de mudas de espécies nativas: substrato, nutrição, sombreamento e fertilização. In: GONÇALVES, J. L. M.; BENEDETTI, V. (Eds.) Nutrição e fertilização florestal. Piracicaba: IPEF, 2000. p.309-350.

IBAMA - Portaria $N^{\circ} 37-\mathbf{N}, 3$ de abril de 1992. Disponível em: http:// www.cetesb.sp.gov.br/licenciamentoo/legislacao/ federal/portarias/1992_Port_IBAMA_37.pdf.

LORENZI, H. Árvores brasileiras: Manual de identificação e cultivo de plantas arbóreas nativas do Brasil. Nova Odessa: Plantarum, 1992. 352p.

LUCHESE, E. B.; FAVERO, L. O. B.; LENZI, E. Fundamentos da química do solo. Rio de Janeiro: Freitas Bastos, 2001. p.111.
MARQUES, V. B. et al. Efeito de fontes e doses de nitrogênio no crescimento de mudas de sabiá (Mimosa caesalpiniaefolia Benth.) Scientia Forestalis, n. 71, p. 77-85, 2006a.

MARQUES, V. B. et al. Efeito de fontes e doses de nitrogênio sobre o crescimento inicial e qualidade de mudas de jacarandá-da-bahia (Dalbergia nigra (Vell.) Fr. All. ex Benth.). Revista Árvore, v.30, n.5, p.725-735, 2006 b.

MELLO, F. A. F. et al. Fertilidade do solo. São Paulo: Nobel, 1983. p.45-72.

MENDONÇA, A. V. R. et al. Exigências nutricionais de Myracrodruon urundeuva Fr. All (Aroeira do Sertão). Revista Cerne, v. 5, n. 2, p.65-75, 1999.

MIELNICZUK, J. et al. Manejo de solo e culturas e sua relação com os estoques de carbono e nitrogênio do solo. In: CURI, N. et al. (Eds.). Tópicos em ciência do solo. Viçosa, MG: Sociedade Brasileira de Ciência do Solo, 2003. v.3. p.209-248.

NAMBIAR, E. K. S. Plantation forests: their scope and perspective $\mathrm{n}$ plantation nutrition. In: BOWER, G. D.; NAMBIAR, E. K. S. (Eds.) Nutrition of plantation forest. London: Academic Press, 1989. p.1-15.

RAIJ, B.van; QUAGGIO, J. A. Métodos de análises de solos para fins de fertilidade. Campinas: Instituto Agronômico de Campinas, 1983. 31p. (Boletim Técnico, 81).

SALATI, E.; SANTO, Â. A.; KLABIN, I. Temas ambientais relevantes. Estudos Avançados, v.20, n.56, p.107-127, 2006.

SOUZA, E. R. B. et al. Crescimento e sobrevivência de mudas de cagaiteira (Eugenia dysenterica DC) nas condições do cerrado. Revista Brasileira de Fruticultura, v. 24, n. 2, p.491-495, 2002.

TEIXEIRA, L. A. J.; WILLIAM, N.; RUGGIERO, C. Alterações em alguns atributos químicos do solo decorrentes da irrigação e adubação nitrogenada e potássica em bananeira após dois ciclos de cultivo. Revista Brasileira de

Fruticultura, v.23, n. 3, p. 684-689, 2001.

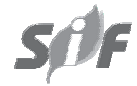

Revista Árvore, Viçosa-MG, v.35, n.3, p.401-411, 2011 
